\title{
Magnetic field transport in propagating thermonuclear burn
}

Cite as: Phys. Plasmas 28, 032705 (2021); https://doi.org/10.1063/5.0040161

Submitted: 10 December 2020 • Accepted: 09 February 2021 • Published Online: 05 March 2021

B. Appelbe, (D) A. L. Velikovich, M. Sherlock, et al.

(1)

\section{ARTICLES YOU MAY BE INTERESTED IN}

Constraining computational modeling of indirect drive double shell capsule implosions using experiments

Physics of Plasmas 28, 032709 (2021); https://doi.org/10.1063/5.0040290

Time resolved ablator areal density during peak fusion burn on inertial confinement fusion implosions

Physics of Plasmas 28, 032701 (2021); https://doi.org/10.1063/5.0038206

Investigation of heat transport using directly driven gold spheres

Physics of Plasmas 28, 032707 (2021); https://doi.org/10.1063/5.0040320 


\title{
Magnetic field transport in propagating thermonuclear burn
}

\author{
Cite as: Phys. Plasmas 28, 032705 (2021); doi: 10.1063/5.0040161 \\ Submitted: 10 December 2020 - Accepted: 9 February 2021 . \\ Published Online: 5 March 2021
}

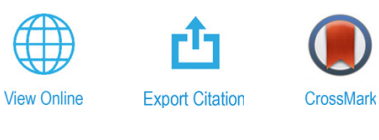

\section{B. Appelbe, ${ }^{1, a)}$ (D) A. L. Velikovich, ${ }^{2}$ (D) M. Sherlock, ${ }^{3}$ C. Walsh, ${ }^{3}$ (D) A. Crilly, ${ }^{7}$ (D) S. O' Neill, ${ }^{7}$ (D) and J. Chittenden ${ }^{7}$}

\author{
AFFILIATIONS \\ T'The Centre for Inertial Fusion Studies, The Blackett Laboratory, Imperial College, London SW7 2AZ, United Kingdom \\ ${ }^{2}$ Plasma Physics Division, Naval Research Laboratory, Washington, DC 20375, USA \\ ${ }^{3}$ Lawrence Livermore National Laboratory, P.O. Box 808, Livermore, California 94551-0808, USA
}

${ }^{a)}$ Author to whom correspondence should be addressed: bappelbe@ic.ac.uk

\begin{abstract}
High energy gain in inertial fusion schemes requires the propagation of a thermonuclear burn wave from hot to cold fuel. We consider the problem of burn propagation when a magnetic field is orthogonal to the burn wave. Using an extended-MHD model with a magnetized $\alpha$ energy transport equation, we find that the magnetic field can reduce the rate of burn propagation by suppressing electron thermal conduction and $\alpha$ particle flux. Magnetic field transport during burn propagation is subject to competing effects: the field can be advected from cold to hot regions by ablation of cold fuel, while the Nernst and $\alpha$ particle flux effects transport the field from hot to cold fuel. These effects, combined with the temperature increase due to burn, can cause the electron Hall parameter to grow rapidly at the burn front. This results in the formation of a self-insulating layer between hot and cold fuel, which reduces electron thermal conductivity and $\alpha$ transport, increases the temperature gradient, and reduces the rate of burn propagation.
\end{abstract}

(C) 2021 Author(s). All article content, except where otherwise noted, is licensed under a Creative Commons Attribution (CC BY) license (http:// creativecommons.org/licenses/by/4.0/). https://doi.org/10.1063/5.0040161

\section{INTRODUCTION}

The ultimate goal of inertial fusion energy is to produce high energy gain, in which the energy liberated in thermonuclear reactions is orders of magnitude larger than the energy of the driver used to compress the fusion fuel. High energy gain can be achieved by using the driver energy to ignite a small fraction of the fuel, the hotspot, which then drives a propagating thermonuclear burn wave into a surrounding layer of dense, cold fuel. ${ }^{1,2}$ The burn wave consists of significant energy transport due to $\alpha$ particles, electron thermal conduction, and radiative processes.

Magnetic fields are a central feature of a broad class of inertial fusion schemes referred to as magneto-inertial fusion (MIF). ${ }^{4}$ In MIF, a magnetic field is applied to the fuel during the compression phase. This magnetic field provides magnetothermoinsulation ${ }^{5}$ during the compression phase by reducing electron thermal conduction losses from hot plasma. This reduces the implosion velocity required to reach the ignition temperature. The magnetic field also confines $\alpha$ particles within the fuel during thermonuclear burn but with a Larmor radius that is significantly larger than that of the thermal electrons.

A number of MIF schemes under current investigation, such as MagLIF at Sandia National Laboratories, ${ }^{6,7}$ aim to achieve volumetric thermonuclear burn. Volumetric burn schemes are designed to heat all the fuel to fusion temperatures during the compression phase. If the fuel ignites, then a net energy gain can be achieved without the need for thermonuclear burn propagation (even though the theoretical maximum energy gain is smaller for volumetric burn compared with propagating burn). For volumetric burn schemes, it is desirable to have a magnetic field, which maximizes the magnetothermoinsulation, as long as the magnetic pressure remains small relative to thermal pressure.

In the case of MIF schemes involving propagating burn, such as high gain MagLIF ${ }^{2}$ and magnetized indirect-drive inertial confinement fusion (ICF), ${ }^{8,9}$ the magnetothermoinsulation effect is required during the compression phase to aid the formation of an igniting hotspot. However, the role of the magnetic field during the propagating burn phase is less clear. The suppression of thermal conduction and $\alpha$ particle transport reduces the rate of burn propagation into the cold fuel. ${ }^{2,10,11}$ This can limit the energy gain achieved before the target disassembles. While some magnetic confinement of $\alpha$ particles during burn propagation could be desirable, to allow for lower areal densities, it is clear that a magnetic field, which is too large, could be detrimental to the achievable yield. This question of how a magnetic field affects thermonuclear burn propagation motivates the present work. In 
addition to propagating burn MIF schemes, this question may also be relevant to conventional ICF since simulations have shown that the Biermann battery mechanism ${ }^{12}$ can generate magnetic fields at the surface of the hotspot in perturbed ICF implosions. ${ }^{13}$

In this work, we study magnetic field dynamics in a propagating burn wave and the effect of the magnetic field on the evolution of burn propagation. The principal novelty of the work is our detailed treatment of the magnetic field transport. We utilize an MHD model (described in detail in Sec. II) in which the induction equation includes advection, resistive diffusion, electron temperature gradients (the Nernst effect), and the flux of $\alpha$ particles. The last term is typically not included in simulations of MIF schemes. We find that the various terms in the induction equation result in competing dynamics of the magnetic field at a propagating burn front: the field is advected from cold to hot regions as the burn wave causes ablation of cold fuel, while temperature gradients and $\alpha$ particle fluxes transport the field in the opposite direction. These dynamics have a significant effect on the shape and rate of propagation of the burn wave.

The magnetic field profile at the onset of burn in MIF is determined by the implosion phase, and there has been a number of studies showing the complex field transport occurring in that phase. ${ }^{14-17}$ For simplicity, we do not study the implosion phase in the current work. Instead, we take as our starting point a region of hot fuel that is undergoing significant $\alpha$ heating adjacent to a cold fuel layer. In order to elucidate the field dynamics during burn, we focus on three simple cases for the initial magnetic field profile: a spatially uniform field, a spatially uniform electron Hall parameter, and a magnetic field that is nonzero only in a small region at the burn front. The results for each of these cases are discussed in Sec. III.

This is not an exhaustive search of magnetic field strengths and profiles, but the cases considered show that there is a complex interplay between the magnetic field dynamics and the evolution of the propagating burn wave. The suppression of thermal conduction and $\alpha$ transport by the magnetic field depends on both the magnitude of the magnetic field and the collisionality of the plasma, where the relevant metric is the Hall parameter. As thermonuclear burn heats the plasma, collisionality decreases rapidly. Combining this with the magnetic field transport effects leads to enhanced suppression of energy transport from hot to cold fuel. In our case studies, we identify scenarios in which a self-insulating magnetized layer can form between hot and cold fuel due to feedback between decreasing collisionality in the burn wave and the magnetic field transport. This self-insulating layer significantly reduces thermal conductivity and $\alpha$ transport across it and leads to the development of large temperature gradients. In Sec. IV, we formulate an equation for the growth rate of the Hall parameter in order to better understand the conditions, leading to formation of the self-insulating layer. This equation illustrates, inter alia, the dependence of the growth rate on the Hall parameter value, $\alpha$ heating, and the temperature and $\alpha$ energy density profiles.

Finally, we present some conclusions in Sec. V. While recent theoretical $^{15}$ and experimental ${ }^{7}$ work has demonstrated the importance of magnetic field transport during the implosion phase, our results show that field transport effects during the burn phase are also of interest.

\section{MODEL OUTLINE}

Our model is a one-dimensional, planar, classical DT plasma consisting of semi-infinite regions of cold and hot fuel, separated by a smooth transition region with an orthogonal magnetic field. This idealized geometry is not intended to model a specific MIF scheme but instead allows us to study how thermonuclear burn propagates from hot to cold fuel. We consider scenarios in which burn propagation and flow speeds are subsonic, allowing us to assume that the plasma remains isobaric, and the plasma beta is large. The system is governed by an equation of continuity, the isobaric condition, an induction equation, and energy equations for the fuel and $\alpha$ particles as follows:

$$
\begin{aligned}
& \frac{\partial n}{\partial \hat{t}}+\frac{\partial}{\partial \hat{x}}(n \hat{u})=0, \\
& \frac{\partial}{\partial \hat{x}}\left(2 n T+\frac{B^{2}}{2 \mu_{0}}\right)=0, \\
& \frac{\partial B}{\partial \hat{t}}+\underbrace{\frac{\partial}{\partial \hat{x}}(\hat{u} B)}_{\text {advect. }}=\frac{\partial}{\partial \hat{x}}[\underbrace{\hat{\alpha} \frac{\partial B}{\partial \hat{x}}}_{\text {res. diff. }}+(\underbrace{\hat{\beta} \frac{\partial \ln T}{\partial \hat{x}}}_{\text {Nernst }}+\underbrace{\hat{\gamma} \frac{\partial \ln \mathcal{E}_{\alpha}}{\partial \hat{x}}}_{\alpha-\text { e coll. }}) B], \\
& 3 n \frac{D T}{D \hat{t}}+2 n T \frac{\partial \hat{u}}{\partial \hat{x}}=\frac{\partial}{\partial \hat{x}}[\underbrace{\hat{\kappa} \frac{\partial T}{\partial \hat{x}}}_{\text {cond. }}+\underbrace{\hat{\beta} \frac{B}{\mu_{0}} \frac{\partial B}{\partial \hat{x}}}_{\text {Ettings. }}]+\underbrace{\hat{q}_{\alpha}}_{\alpha \text { heat. }}-\underbrace{\hat{P}}_{\text {brem. }}, \\
& \frac{D \mathcal{E}_{\alpha}}{D \hat{t}}+\frac{5}{3} \mathcal{E}_{\alpha} \frac{\partial \hat{u}}{\partial \hat{x}}=\underbrace{\frac{\partial}{\partial \hat{x}}\left(\hat{\delta}_{\mathcal{E}} \frac{\partial \mathcal{E}_{\alpha}}{\partial \hat{x}}\right)}_{\alpha \text { energy diff. }}-\hat{q}_{\alpha}+\underbrace{\hat{Q}}_{\text {reac. }} \text {, }
\end{aligned}
$$

where $\hat{t}, \hat{x}$, and $\hat{u}$ represent the dimensionless time, position, and fluid velocity of the fuel and

$$
\frac{D}{D \hat{t}}=\frac{\partial}{\partial \hat{t}}+\hat{u} \cdot \frac{\partial}{\partial \hat{x}}
$$

is the convective derivative. The normalizing constants are $L_{T}$, representing the width of the transition region between hot and cold fuel and $\mathcal{T}_{h}$, the stopping time for a $3.45 \mathrm{MeV} \alpha$ particle in the initial hot fuel. Other variables include the fuel density, $n$, magnetic field, $B$, fuel temperature, $T$, and energy density of $\alpha$ particles, $\mathcal{E}_{\alpha}$. SI units are used for quantities that have not been made dimensionless.

The induction, (3), and fuel energy, (4), equations contain classical magnetized transport coefficients for resistivity, thermoelectricity, and thermal conductivity, ${ }^{18}$

$$
\begin{gathered}
\hat{\alpha}=\frac{\mathcal{T}_{H}}{L_{T}^{2}} \frac{m_{e}}{\mu_{0} e^{2} n \tau_{e i}} \alpha_{\perp}^{c}, \quad \hat{\beta}=\frac{\mathcal{T}_{H}}{L_{T}^{2}} \frac{\tau_{e i} T}{m_{e}} \frac{\beta_{\curlywedge}}{\chi_{e}}, \\
\hat{\kappa}=\frac{\mathcal{T}_{H}}{L_{T}^{2}} \frac{n T \tau_{e i}}{m_{e}}\left(\kappa_{\perp e}^{c}+\sqrt{\frac{2 m_{e}}{m_{i}}} \kappa_{\perp i}^{c}\right) .
\end{gathered}
$$

The coefficients $\alpha_{\perp}^{c}, \beta_{\wedge}, \kappa_{\perp e}^{c}$, and $\kappa_{\perp i}^{c}$ are functions of electron Hall parameter, $\chi_{e}=e B \tau_{e i} / m_{e}$, where $\tau_{e i}=6 \varepsilon_{0}^{2} \sqrt{2 \pi^{3} m_{e}} T^{\frac{3}{2}} /\left(e^{4} \ln \Lambda_{e i} n\right)$. We use the tabulated values of Epperlein and Haines ${ }^{19}$ for these coefficients. The chosen geometry of our problem means that the Hall term $^{20}$ and Biermann battery term ${ }^{12}$ do not need to be considered in the induction equation.

The final term on the rhs of (3) represents the collisionally induced current arising from the interaction of the $\alpha$ particle flux with 
the thermal electrons. Similar fast ion effects have previously been studied for collisionless plasmas in current drive in tokamaks, ${ }^{21}$ cosmic rays in astrophysical plasmas, ${ }^{22}$ and relativistic particles in laserplasma interactions. ${ }^{23}$ Here, we follow the procedure of Appelbe et al. $^{24}$ to include these effects in a collisional, magnetized plasma.

An analogy may be drawn between the Nernst and $\alpha$-e collisional terms in (3). The Nernst effect is a result of the effect of a magnetic field on the thermal force. ${ }^{18}$ Electrons being driven by a temperature gradient and experiencing friction due to the background ions are deflected by a magnetic field that is orthogonal to the temperature gradient, generating an electron current orthogonal to both. In the case of the $\alpha$-e collisional term, the electron current is instead driven by a flux of $\alpha$ particles. Both the electron temperature gradient and the $\alpha$ flux are themselves dependent on the magnetic field. The interplay between the temperature gradient, magnetic field, and driven electron current has been well studied ${ }^{15}$ and is often included in simulations. However, the equivalent interplay between the magnetic field, the $\alpha$ flux, and the driven electron current has not received such attention.

In our geometry, the $\alpha$-e collisional effect can be expressed as

$$
\begin{gathered}
\hat{\gamma}=\mathcal{E}_{\alpha}\left(\frac{\partial \mathcal{E}_{\alpha}}{\partial \hat{x}}\right)^{-1} \frac{Z_{\alpha}}{n}\left(\gamma_{\perp} F_{\perp}+\gamma_{\wedge} F_{\wedge}\right), \\
\gamma_{\perp}=-j_{\perp}^{\nu}\left(1+\frac{\alpha_{\wedge}^{c}}{\chi_{e}}\right)+j_{\wedge}^{\nu} \frac{\alpha_{\perp}^{c}}{\chi_{e}}, \\
\gamma_{\wedge}=j_{\wedge}^{\nu}\left(1+\frac{\alpha_{\curlywedge}^{c}}{\chi_{e}}\right)+j_{\perp}^{\nu} \frac{\alpha_{\perp}^{c}}{\chi_{e}},
\end{gathered}
$$

where $F_{\perp}, F_{\wedge}$ are components of $\alpha$ particle flux in the directions of burn propagation and orthogonal to burn propagation and $B$ field, respectively, and $j_{\perp}^{\nu}, j_{\wedge}^{\nu}$ are functions of $\chi_{e}{ }^{24}$

The $\alpha$ particle fluxes can be estimated from the gradients of $\mathcal{E}_{\alpha}$ and $\alpha$ particle diffusion coefficients as follows: ${ }^{25}$

$$
\begin{gathered}
F_{\perp, \wedge}=-\frac{\hat{\delta}_{p \perp, \wedge}}{\left\langle E_{\alpha}\right\rangle} \frac{\partial \mathcal{E}_{\alpha}}{\partial \hat{x}}, \\
\hat{\delta}_{p \perp}=\frac{\mathcal{T}_{h}}{L_{T}^{2}} \frac{E_{\alpha 0} \tau_{\alpha}}{3 m_{\alpha}\left(1+\chi_{\alpha}^{2}\right)}, \quad \hat{\delta}_{p \wedge}=\chi_{\alpha} \hat{\delta}_{p \perp},
\end{gathered}
$$

where $\left\langle E_{\alpha}\right\rangle \approx E_{\alpha 0} / 2$ is the mean $\alpha$ particle energy.

The $\alpha$ energy transport equation (5) represents a single group diffusion approximation. ${ }^{25}$ It contains terms for magnetized diffusion, slowing on electrons and a source due to DT reactions, ${ }^{26}$

$$
\begin{gathered}
\hat{\delta}_{\mathcal{E}}=\frac{\mathcal{T}_{h}}{L_{T}^{2}} \frac{E_{\alpha 0} \tau_{\alpha}}{m_{\alpha}\left(9+\chi_{\alpha}^{2}\right)}, \quad \hat{\nu}=\frac{\mathcal{T}_{h}}{\tau_{\alpha}}, \quad \hat{Q}=\mathcal{T}_{h} \frac{n^{2}}{4}\langle\sigma v\rangle_{D T} E_{\alpha 0}, \\
\chi_{\alpha}=\frac{Z_{\alpha} e B}{m_{\alpha}} \tau_{\alpha}, \quad \tau_{\alpha}=3 \sqrt{\frac{\pi^{3}}{2 m_{e}}} \frac{m_{\alpha} \varepsilon_{0}^{2}}{e^{4} \ln \Lambda_{\alpha e}} \frac{T^{\frac{3}{2}}}{n}
\end{gathered}
$$

where $\chi_{\alpha}$ is the $\alpha$ Hall parameter, $\tau_{\alpha}$ is the slowing time on electrons, and $E_{\alpha 0}=3.45 \mathrm{MeV}$. Electron and $\alpha$ Hall parameters are related by $\chi_{\alpha} / \chi_{e}=Z_{\alpha} \ln \Lambda_{e i} /\left(4 \ln \Lambda_{\alpha e}\right)$. Finally, the $\alpha$ heating term, $\hat{q}_{\alpha}$, and bremsstrahlung losses, ${ }^{27} \hat{P}$, are given by

$$
\hat{q}_{\alpha}=2 \hat{\nu} \mathcal{E}_{\alpha}, \quad \hat{P}=1.69 \times 10^{-38} n^{2} \sqrt{\frac{T}{e}} \mathcal{T}_{h} \mathrm{~J} \mathrm{~m}^{-3} .
$$

Returning to the induction equation (3), we note that it has been written such that $\hat{\alpha}, \hat{\beta}$, and $\hat{\gamma}$ can all be treated as diffusion coefficients. In the burning plasma regime, the magnetic Lewis number, ${ }^{14}$ defined as $L m=\hat{\kappa} /\left.(3 n \hat{\alpha})\right|_{\chi_{e}=0}$, is large, meaning that thermal diffusivity is much larger than magnetic diffusivity, and so, resistive diffusion plays only a minor role in $B$ field transport. Using (9) for $\alpha$ particle fluxes, we can write (6) more compactly as

$$
\hat{\gamma}=\frac{\mathcal{T}_{h}}{L_{T}^{2}} \frac{Z_{\alpha} \mathcal{E}_{\alpha} E_{\alpha 0} \tau_{\alpha}}{3 m_{\alpha} n\left\langle E_{\alpha}\right\rangle} \underbrace{\left[\frac{-\gamma_{\perp}-\chi_{\alpha} \gamma_{\curlywedge}}{1+\chi_{\alpha}^{2}}\right]}_{\Gamma_{\gamma}},
$$

where the dependency on magnetization is now fully contained in the square brackets, which we now denote as $\Gamma_{\chi}$. We note that even though (7) and (8) contain terms $\propto 1 / \chi_{e}$, the term $\Gamma_{\chi}$ does not diverge at $\chi_{e}=0$ since $\alpha_{\wedge}^{c}, j_{\wedge}^{\nu}, F_{\wedge} \rightarrow 0$ as $\chi_{e} \rightarrow 0$.

The $\Gamma_{\chi}$ term is compared with the dependence of $\hat{\beta}$ (which determines the magnitude of the Nernst effect) on $\chi_{e}$ in Fig. 1. Comparing the unmagnetized components of $\hat{\beta}$ and $\hat{\gamma}$ gives

$$
\frac{\hat{\gamma}}{\hat{\beta}} \sim \frac{E_{\alpha 0}}{6 T} \frac{n_{\alpha}}{n} \frac{\ln \Lambda_{e i}}{\ln \Lambda_{\alpha e}} \Gamma_{\chi}\left(\frac{\chi_{e}}{\beta_{\curlywedge}}\right),
$$

where $n_{\alpha}$ is the number density of nonthermalized $\alpha$ particles. We can assume that the ratio of Coulomb logarithms is of order unity and $E_{\alpha 0} /(6 T) \sim 10^{2}$ for an igniting plasma. Therefore, a relative density of $\alpha$ particles of $n_{\alpha} / n \sim 10^{-2}$ could be sufficient for the $\hat{\gamma}$ term to dominate.

It is also worth noting that $\hat{\beta} \geq 0$ for all values of $\chi_{e}$. This is because in a plasma, the friction force exerted by background ions is lower for faster electrons. However, $\Gamma_{\chi}<0$ for very large and very small values of $\chi_{e}$. This is due to $j_{\perp}^{\nu}<0$ at small values of $\chi_{e}$, while for large values, the $-\chi_{\alpha} \gamma_{\wedge}$ term dominates.

The system of Eqs. (1) - (5) is solved using an implicit Lagrangian model in order to accurately resolve the steep gradients that exist at the burn front. ${ }^{14,15}$ We use the isobaric condition, (2), in place of a momentum equation for simplicity as our principal interest is the transport processes occurring in (3)-(5). However, this limits our studies to burn propagation regimes driven by deflagration rather than detonation.

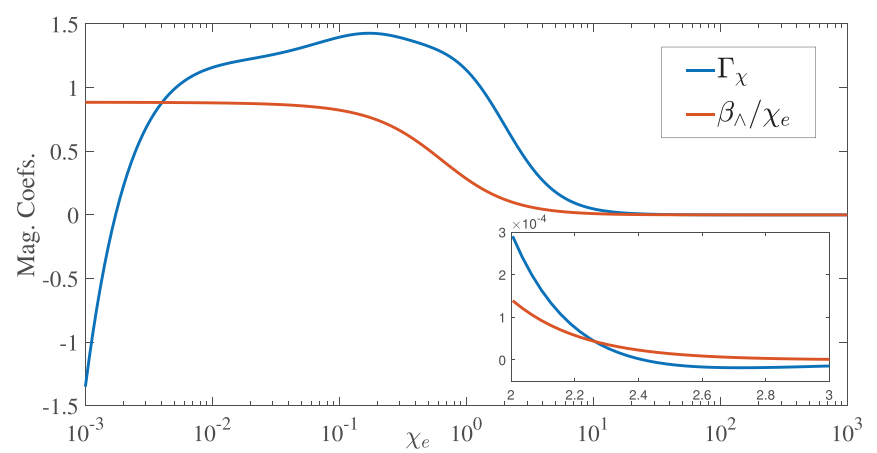

FIG. 1. The variation of the diffusion coefficients $\hat{\beta}$ and $\hat{\gamma}$ with $\chi_{e}$, where we assume $\chi_{\alpha}=\chi_{e} / 2$. Note that $\beta_{\wedge} / \chi_{e} \geq 0 \forall \chi_{e}$, while $\Gamma_{\chi}<0$ for $\chi_{e} \ll 1$ and $\chi_{e} \gg 1$. For $\chi_{e}=0$, we have $\Gamma_{\chi} \approx-1.7$. The scale of the horizontal axis of the inset plot represents $\log _{10} \chi_{e}$ 


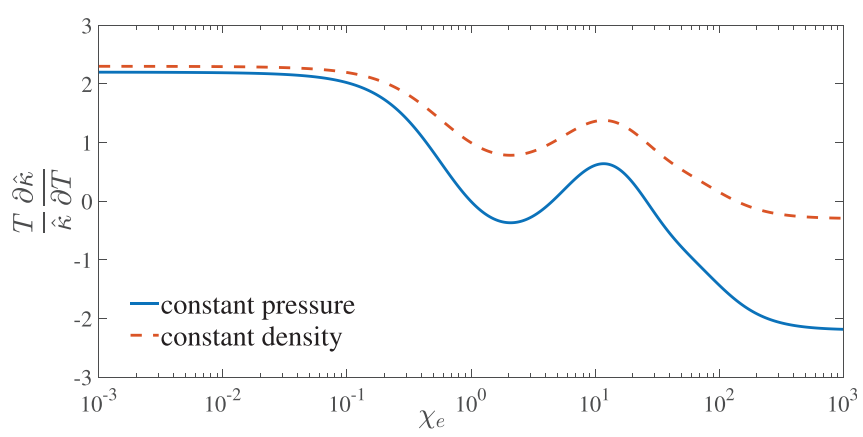

FIG. 2. The normalized rate of change of thermal conductivity with temperature as a function of $\chi_{e}$, under the assumption of either constant density ( $n$ is fixed) or constant thermal pressure ( $n T$ is fixed). The peak near $\chi_{e} \approx 10$ corresponds to the point at which ion thermal conductivity dominates over that of the electrons (see Fig. 9).

Finally, Fig. 2 shows how thermal conductivity $\hat{\kappa}$ varies with temperature, appropriately normalized by $\hat{\kappa}$ and $T$. At $\chi_{e}=0$, this quantity has a value of $\approx 2.5$ due to the scaling of Spitzer thermal conductivity $\propto T^{5 / 2}$, neglecting variations in $\ln \Lambda_{e i}$. However, for $\chi_{e} \gtrsim 0.1$, this scaling is significantly reduced with regions near $\chi_{e} \approx 1$ and $\chi_{e} \gtrsim 20$ for which it is negative, indicating that increasing temperature will cause a decrease in thermal conductivity. This is because increasing temperature causes $\chi_{e}$ to increase and the suppression of thermal conduction due to increasing magnetization outweighs the $T^{5 / 2}$ scaling of Spitzer conductivity.

This relationship between thermal conductivity and temperature means that a magnetized plasma can be self-insulating, with increasing temperature, causing a reduction in the transport of thermal energy from hot to cold plasma. This mechanism will be illustrated in more detail in Sec. III where we will find that the transport of the magnetic field can enhance the effect. Figure 2 shows results for a plasma that is assumed to have both constant density (zero fluid motion) and constant pressure. Both results follow a similar trend, suggesting that the self-insulating mechanism may occur even when the isobaric assumption is relaxed.

\section{BURN PROPAGATION DYNAMICS FOR VARIOUS B PROFILES}

In this section, we apply the model to a variety of different initial $B$ field profiles. In each case, the initial temperature profile is chosen to be sigmoidal,

$$
T_{0}(\hat{x})=T_{c}+\left(T_{h}-T_{c}\right)(1+\exp (-10 \hat{x}))^{-1},
$$

where $T_{c}\left(T_{h}\right)$ is the temperature of the cold (hot) fuel. The factor of 10 ensures that $98 \%$ of the temperature change takes place within a region of unity length. The normalizing constant, $L_{T}$, is the burn front scale length. Its value is chosen to be some multiple of the stopping distance of a $3.45 \mathrm{MeV} \alpha$ particle at the conditions of the midpoint of the initial temperature profile.

\section{A. Uniform $B$ field}

We begin with the case of a $B$ field whose strength is initially uniform across the burn front. Since $B$ is constant, $\chi_{e} \propto \tau_{e i}$, and the initial

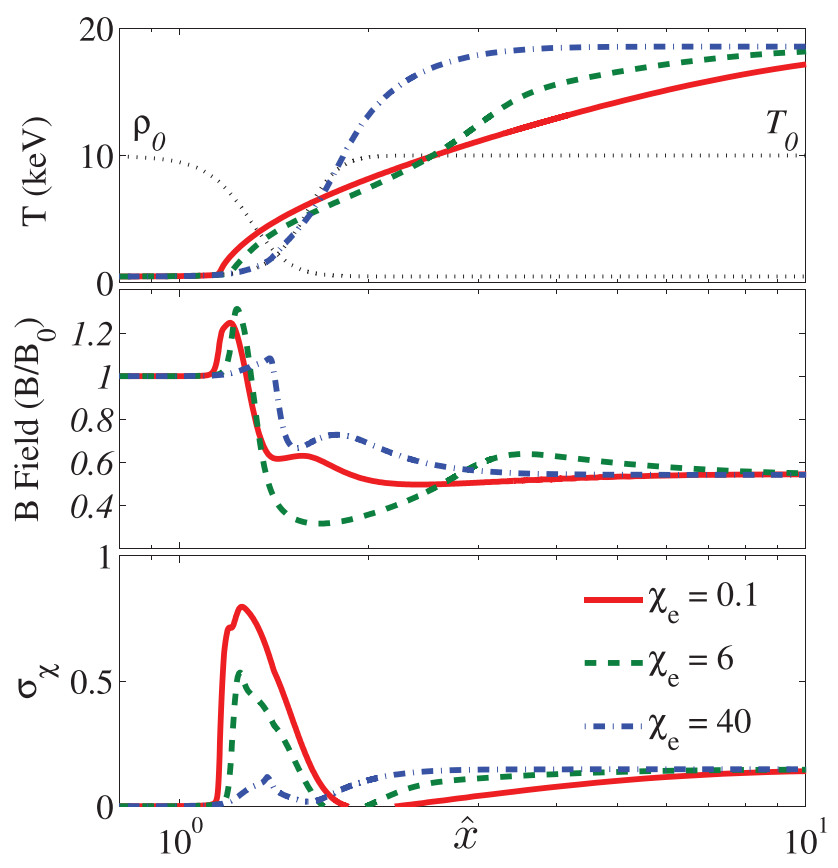

FIG. 3. Profiles at $\hat{t}=5 \mathcal{T}_{h}$ for the initially uniform $B$ field with $\chi_{e}=0.1,6,40$ in the hot fuel. Top: Temperatures. Initial temperature, $T_{0}$, and density, $\rho_{0}$, are also shown. Middle: $B$ fields where $B_{0} \approx 80,500,3500 T$ for $\chi_{e}=0.1,6,40$, respectively. Bottom: Growth rates of $\chi_{e}$. The parameter values of $T_{c}=500 \mathrm{eV}, T_{h}=10 \mathrm{keV}, n_{h}$ $=10^{31} \mathrm{~m}^{-3}$, and $L_{T}=0.3$ were used. Note that the isobaric condition means that the self-heating hot fuel is allowed to freely expand, resulting in $B$ decreasing uniformly in the hot fuel as $T$ increases.

value of $\chi_{e}$ will vary significantly across the burn front with the largest value in the hot fuel. Figure 3 shows results for three different initial values of $B$ in which $L_{T}$ is 0.3 times the $\alpha$ stopping distance, corresponding to $\sim 15 \mu \mathrm{m}$. The initial values of $\chi_{e}$ in the hot fuel were $0.1,6,40$, corresponding to $B_{0} \approx 80,500,3500 \mathrm{~T}$. The corresponding values in the cold fuel were a factor of $\sim 800$ smaller. We note that at $\chi_{e}=0.1,6,40$, the electron thermal conductivity is a factor of $\sim 1,10^{-1}, 10^{-3}$ lower than its unmagnetized value. The maximum mean free path of thermal electrons in this system is $\lambda_{e i} \approx 0.009 L_{T}$, ensuring validity for our MHD model.

As burn propagates, the evolution of the burn front is highly sensitive to the $B$ field. The $\chi_{e}=0.1$ case (which closely resembles an unmagnetized burn front) develops a temperature profile that is far less steep than the more magnetized cases and propagates furthest into the cold fuel. However, this burn front also contains a localized region of steep temperature gradient near the cold fuel, which we refer to as the foot of the burn front. This is caused by rapid $\alpha$ heating of the cold, dense fuel. In the more magnetized cases, the burn propagates less far due to suppression by the magnetic field of thermal conduction and $\alpha$ transport from hot to cold fuel.

The transport of the $B$ field during burn is also dependent on $\chi_{e}$. The middle diagram of Fig. 3 illustrates competing transport effects. The Nernst and $\alpha$ flux effects transport the $B$ field from hot to cold plasma. This is most clearly evidenced by the spikes at the foot of the burn front where temperature gradients rapidly increase. This is similar to the Nernst waves that have previously been studied in MagLIF 
and magnetized laser ablation. ${ }^{15,28}$ Magnetic resistivity limits the sharpness of these spikes. Meanwhile, ablation of cold fuel by the burn front leads to the $B$ field being advected from cold to hot fuel. Evidence of the ablative effect is shown in the $\chi_{e}=6$ case, where a dip in the $B$ field centered at $\hat{x}=1.7$ coincides with a shallower temperature gradient.

The dynamics at an evolving burn front can cause both compression and rarefaction of a magnetic field. Due to the isobaric condition and the increasing temperature of the plasma, advection does not cause any compression of the $B$ field, only rarefaction. However, the Nernst effect also contributes to rarefaction of the $B$ field in regions behind the compression wave. This effect has previously been observed in the compression phase of $\mathrm{MagLIF}^{6}$ and will be more clearly shown in Fig. 5.

The bottom diagram of Fig. 3 shows profiles for $\sigma_{\chi}$, the exponential growth rate for $\chi_{e}$ at $\hat{t}=5 \mathcal{T}_{h}$. The value of $\chi_{e}$ can increase due to both heating of the plasma (which increases $\tau_{e i}$ ) and $B$ field transport. In the cases of $\chi_{e}=0.1$ and 6 , the peak values of $\sigma_{\chi}$ occur near the foot of the burn front and are significantly larger than the $\sigma_{\chi}$ values in the hot fuel. In the $\chi_{e}=40$ case, the temperature at the foot of the burn front increases at a slower rate, and so, the value of $\sigma_{\chi}$ is approximately the same as that in the hot fuel.

The effects of these transport phenomena on burn propagation are illustrated in Fig. 4. There is a significant drop in the rate of burn propagation as the initial value of $\chi_{e}$ increases. The hot fuel is insulated from the cold fuel, and since $\sigma_{\chi}$ is large at the burn front, this insulating effect increases as burn evolves. A similar behavior is found for a wide range of initial parameters $\left(n_{h} \sim\left[10^{28}, 5 \times 10^{31}\right] \mathrm{m}^{-3}\right.$ and $\left.T_{h} \gtrsim 5 \mathrm{keV}\right)$.

We conclude this subsection with the results of an integrated simulation (see Fig. 5), carried out using the Chimera code, ${ }^{3}$ which includes the radiation transport, extended-MHD, and $\alpha$ transport package. However, the $\alpha-e$ collisional term has not yet been included in this model. These simulations used a similar setup and initial conditions to those shown in Fig. 3 but with an initial value of $\chi_{e} \approx 1$ in the hot fuel.

The Nernst term causes a significant spike in the $B$ field at the burn front, with a corresponding spike in $\chi_{e}$, by compressing the $B$ field. A comparison of the $B$ field profiles with and without Nernst, shown in the middle panel, demonstrates how the Nernst also causes

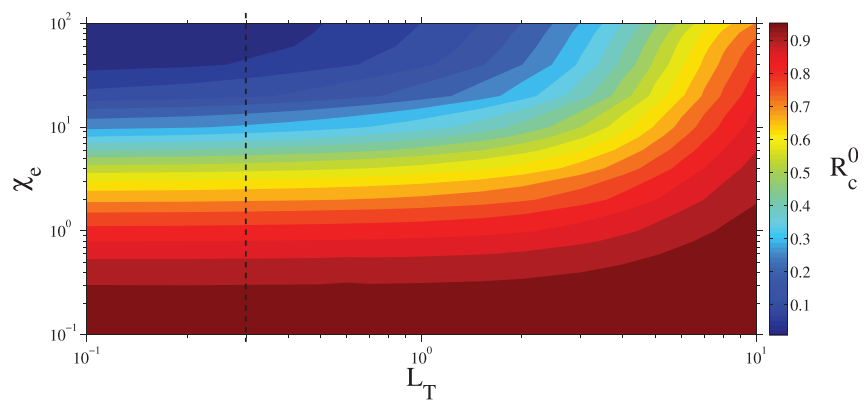

FIG. 4. Ratio of the burn rate in cold fuel to the unmagnetized burn rate at $\hat{t}=5 \mathcal{T}_{h}$. Cold fuel is defined as all fuel with an initial temperature less than $\left(T_{c}+T_{h}\right) / 2$. The $B$ field is initially uniform, and $\chi_{e}$ denotes the initial value in the hot fuel. The values of $T_{c}, T_{h}$, and $n_{h}$ are as in Fig. 3 . The dashed black line indicates $L_{T}=0.3$, the cases shown in Fig. 3 .

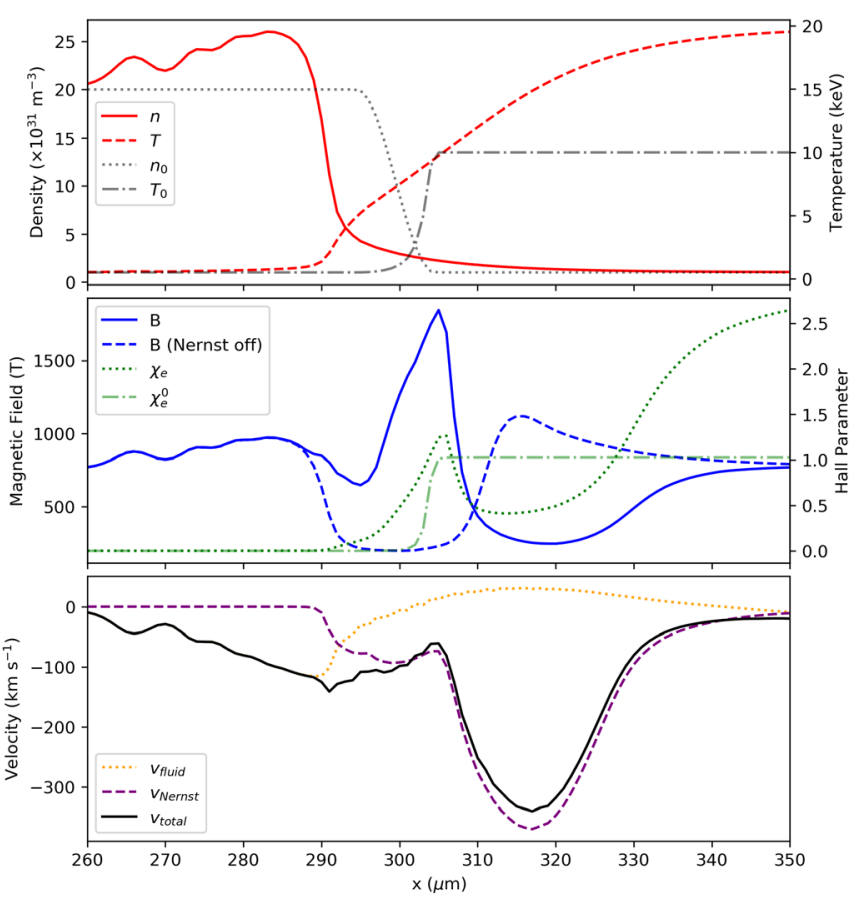

FIG. 5. Results from an integrated simulation with an initially uniform $B$ field. Top: Initial and final profiles of temperature and density. Middle: Final profiles of the $B$ field with and without the Nernst term included and also initial and final $\chi_{e}$ profiles. Bottom: $B$ field advection velocity $\left(v_{\text {fluid }}\right)$, Nernst velocity $\left(v_{\text {Nernst }}\right)$, and their sum.

rarefaction of the $B$ field behind this compression wave. The bottom panel of Fig. 5 shows the velocity components of $B$ field transport with $v_{\text {fluid }}\left(v_{\text {Nernst }}\right)$ equivalent to $\hat{u}(\hat{\beta} \partial \ln T / \partial \hat{x})$ in (3). From this, we can see that the spike in the $B$ field corresponds to a region in which the absolute value of $v_{\text {Nernst }}$ has a local minimum. Although the isobaric condition no longer applies, the evolution of the profiles of $\rho, T$, and $B$ is qualitatively similar to the results obtained from the model outlined in Sec. II and suggests that observations from this model are not unduly compromised by the isobaric condition.

\section{B. Uniform electron Hall parameter}

We next consider the case of a burn front in which $\chi_{e}$ is initially uniform. Figure 6 shows an example of burn propagation where initially $\chi_{e}=0.1$. To achieve the uniformity of $\chi_{e}$, the initial $B$ field needs to be significantly larger in the cold fuel, and so, we choose a parameter value of $n_{h}=10^{30} \mathrm{~m}^{-3}$ to ensure that the magnetic pressure is small compared with thermal pressure.

The top diagram of Fig. 6 illustrates how $\chi_{e}$ grows rapidly in the burn front region due to both increasing temperature and increasing magnetic field. The bottom diagram shows different terms contributing to magnetic field growth in the burn front region.

The final $T$ profile has a distinctive shape with very steep temperature gradients developing at two locations: first, at the foot of the burn pulse where $\alpha$ heating is the largest (see Fig. 7) and second, in the region where $\chi_{e}$ reaches a maximum. This second steep temperature gradient evolves to compensate for the drop in electron thermal conductivity caused by increasing $\chi_{e}$. This temperature profile is 

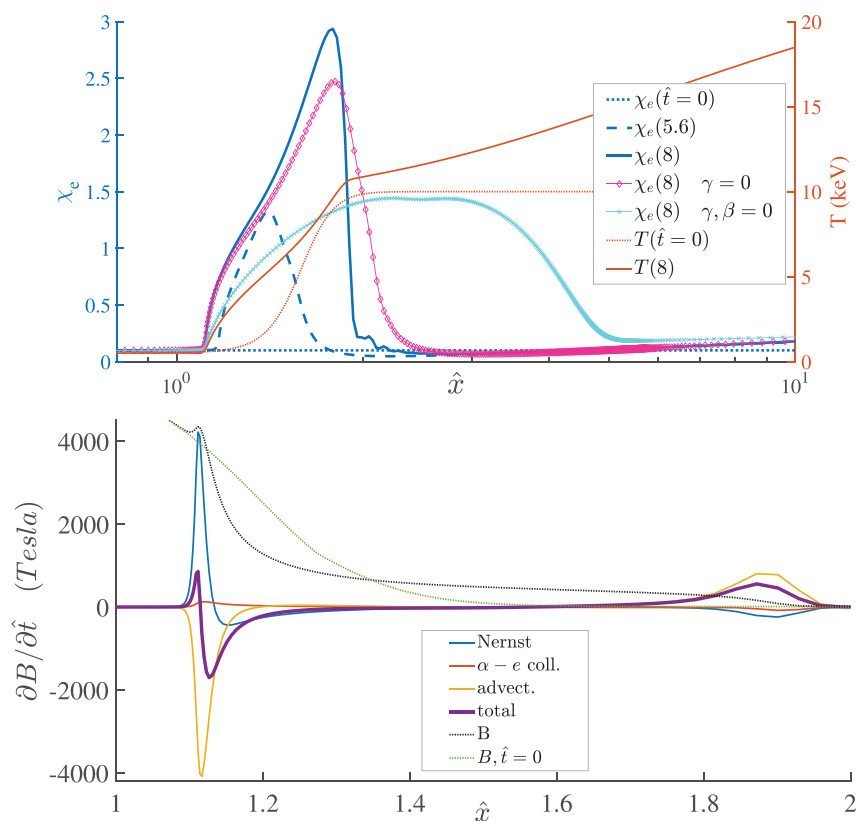

FIG. 6. Top: Evolution of $\chi_{e}$ over a period of $8 \mathcal{T}_{h}$ given an initial value $\chi_{e}=0.1$. Also shown are the initial and final $T$ profiles and the final $\chi_{e}$ profiles when the (i) $\alpha-e$ collision term (labeled $\gamma=0$ ) and (ii) $\alpha-e$ and Nernst terms (labeled $\gamma, \beta=0)$ are neglected. Bottom: Components of the $B$ field growth rate at the final time in the self-insulating region. Also shown are the initial and final $B$ field profiles. Parameters: $T_{c}=500 \mathrm{eV}, T_{h}=10 \mathrm{keV}, n_{h}=10^{30} \mathrm{~m}^{-3}$, and $L_{T}=0.3$.

reminiscent of double ablation front structures, ${ }^{29,30}$ which occur in direct-drive ICF. In that scenario, radiation energy and electron heat flux are absorbed at different locations, leading to the formation of the double ablation front. In our case, the first front is formed by $\alpha$ heating, while the second front is due to the suppression of energy transport.

At the location of the second steep temperature gradient, advection causes $B$ to grow, while $\alpha-e$ collisional and Nernst terms prevent $B$ from penetrating further into the hot fuel, as can be seen from the bottom diagram of Fig. 6 . We describe the region where $\chi_{e}$ grows rapidly as self-insulating since the growth is driven by the dynamics of

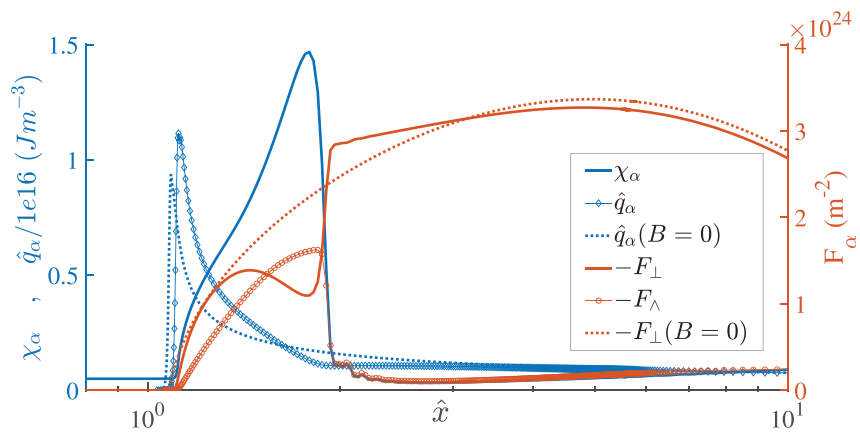

FIG. 7. Profiles of $\chi_{\alpha}, \alpha$ heating, and $\alpha$ flux terms after $8 \mathcal{T}_{h}$. Also shown are the profiles for the case with no $B$ field. The Larmor radius for $\alpha$ particles with energy $\left\langle E_{\alpha}\right\rangle$ at maximum $\chi_{\alpha}$ is $\approx 4$, and its mean free path is $\approx 0.11$. burn propagation. The top diagram of Fig. 6 also shows the final profiles of $\chi_{e}$, which are obtained if these two terms are neglected in the induction equation. Clearly, these terms play a crucial role in establishing the self-insulating region.

Finally, Fig. 7 shows the profiles of quantities related to the $\alpha$ particles. It is found that $\chi_{\alpha}$ evolves in a similar way to $\chi_{e}$ with a spike in $\chi_{\alpha}$ occurring at the burn front. This causes a sharp drop in $F_{\perp}$ and a spike in $F_{\wedge}$ in the self-insulating region, while $\alpha$ heating propagates less far into the cold fuel compared with the unmagnetized case.

\section{Local magnetization}

Integrated simulations of unmagnetized ICF implosions have shown that self-generated $B$ fields can develop at the interface of hot and cold fuel. ${ }^{13}$ In this section, we consider how such a local $B$ field could evolve during burn by assuming that the initial $\chi_{e}$ has a Gaussian profile that is narrower than $L_{T}$. An example of such a scenario is shown in Fig. 8 where the FWHM of the initial $\chi_{e}$ profile is $1 / 3 L_{T}$ with a peak value of $\chi_{e}=1$.

The $B$ field is rapidly advected from cold to hot fuel by the propagating burn. However, the Nernst and $\alpha-e$ collision terms cause this advected $B$ field to maintain a steep front. This coincides with the front of the rapidly growing $\chi_{e}$ profile and a region of very steep temperature gradient. As in the case of initially uniform $\chi_{e}$, the growth in $\chi_{e}$ leads to the formation of a self-insulating layer between hot and cold fuel. This behavior is rather counter-intuitive. Our usual expectation would be that the increasing temperature would increase the rate at which
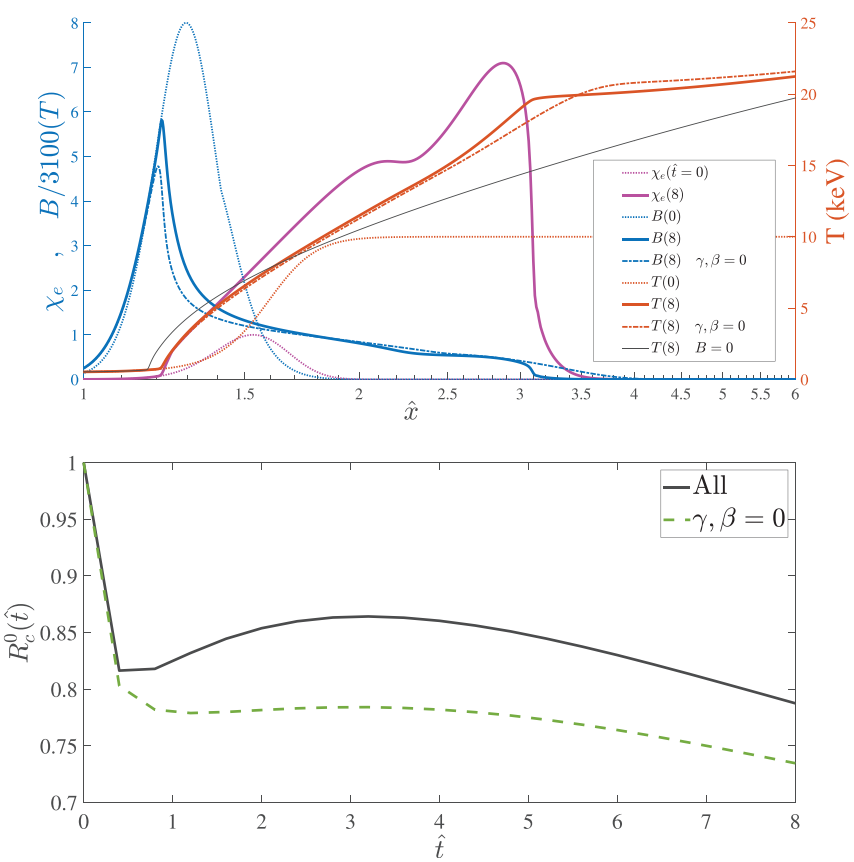

FIG. 8. Top: Profiles of $\chi_{e}, B$, and $T$ at $\hat{t}=0$ and $\hat{t}=8 \mathcal{T}_{h}$ for an initially local $B$ field profile. Also shown are the $B$ and $T$ profiles for when Nernst and $\alpha-e$ collision terms are neglected in the induction equation $(\gamma, \beta=0)$ and the temperature profile for an unmagnetized plasma. Bottom: Ratio of the cold fuel burn rate to the unmagnetized rate as a function of time. The parameter values of $T_{c}=500 \mathrm{eV}, T_{h}=10 \mathrm{keV}, n_{h}=10^{31} \mathrm{~m}^{-3}$, and $L_{T}=0.4=24 \mu \mathrm{m}$ were used. 
transport processes act to smooth out the steep gradients. Instead, we see that the increasing temperature leads to increasing $\chi_{e}$ and a reduction in energy transport such that the gradients become even steeper. This behavior is consistent with the dependence of $\hat{\kappa}$ on $T$ illustrated in Fig. 2.

Figure 8 also shows the reduction in the burn rate of cold fuel as a function of time. We can see from this that even though the majority of fuel is unmagnetized, the growth of $\chi_{e}$ during burn can significantly reduce the rate of burn propagation.

We note that the value of $\kappa_{\perp e}^{c}$, the electron thermal conductivity coefficient, is about an order of magnitude smaller at $\chi_{e}=7$ compared with $\chi_{e}=1$. Theoretical models of burn in unmagnetized $\mathrm{ICF}^{31-33}$ typically do not account for such rapid changes in conductivity, but this may be necessary if the presence of self-generated $B$ fields is verified. Such fields may also alter the ablative stabilization of Rayleigh-Taylor instabilities during burn. ${ }^{34}$

\section{HALL PARAMETER GROWTH RATES}

The formation of a self-insulating layer with two steep temperature gradients during burn propagation is observed across a broad range of simulation parameters and initial $B$ field profiles. It is a result of the Hall parameter growing faster at the burn front than in the hot fuel. Previous work ${ }^{14,35,36}$ has shown that the Nernst and convective transport's joint action makes the effective reduction of magnetic flux and heat losses due to the magnetization scale approximately with the inverse of the Hall parameter. This is similar to the scaling of turbulent diffusive processes occurring in collisionless, strongly magnetized plasmas such as those found in magnetic fusion energy (MFE) systems, as given by the Bohm formula. ${ }^{37}$ Therefore, understanding what causes the Hall parameter to grow at the burn front allows us to quantify how much the transport processes are suppressed.

We can investigate the formation of the self-insulating layer by considering the factors on which $\sigma_{\chi}$ depends. Starting with the definition $\chi_{e}=e B \tau_{e i} / m_{e}$ and assuming an isobaric, high-beta plasma with thermal pressure $P_{0}=2 n T$, we obtain

$$
\sigma_{\chi}=\frac{D \ln \chi_{e}}{D \hat{t}}=\frac{1}{B} \frac{D B}{D \hat{t}}+\frac{g}{T} \frac{D T}{D \hat{t}},
$$

where $g=\left(5-3\left(\ln \Lambda_{e i}\right)^{-1}\right) / 2 \approx 5 / 2$. If we assume that $\chi_{e}$ is uniform, neglect bremsstrahlung losses $\left(\hat{q}_{\alpha} \gg \hat{P}\right)$, and neglect resistive diffusion and Ettingshausen heat flow, then using (3) and (4) results in

$$
\begin{aligned}
\sigma_{\chi}= & \frac{2(g-1)}{5 P_{0}} \hat{q}_{\alpha}+\hat{\beta}\left(\frac{\partial^{2} \ln T}{\partial \hat{x}^{2}}+\left(\frac{\partial \ln T}{\partial \hat{x}}\right)^{2}\right) \\
& +\frac{2(g-1)}{5 P_{0}} T \hat{\kappa}\left(\frac{\partial^{2} \ln T}{\partial \hat{x}^{2}}+(g+1)\left(\frac{\partial \ln T}{\partial \hat{x}}\right)^{2}\right) \\
& +\hat{\gamma}\left(\frac{\partial^{2} \ln \mathcal{E}_{\alpha}}{\partial \hat{x}^{2}}+\left(\frac{\partial \ln \mathcal{E}_{\alpha}}{\partial \hat{x}}\right)^{2}+\frac{\partial \ln T}{\partial \hat{x}} \frac{\partial \ln \mathcal{E}_{\alpha}}{\partial \hat{x}}\right) .
\end{aligned}
$$

The first term on the right-hand-side (the $\alpha$ heating term) will always be positive, but the three remaining terms could be positive or negative, depending on the profiles of $T$ and $\mathcal{E}_{\alpha}$. This equation illustrates why the steep, opposing gradients in $\chi_{e}$ and $T$ are observed in Figs. 6 and 8: locations with $\partial^{2} \ln T / \partial \hat{x}^{2}<0$ lead to $\sigma_{\chi}<0$ and vice versa.
Assuming that the gradient length scales of $T$ and $\mathcal{E}_{\alpha}$ are similar, we can obtain the following estimates of the strengths of different terms:

$$
\begin{gathered}
\frac{2(g-1)}{5 P_{0}} \hat{q}_{\alpha} \approx \mathcal{T}_{H} 6.29 \times 10^{-5} \ln \Lambda_{\alpha e} \frac{\mathcal{E}_{\alpha}}{T_{k e V}^{\frac{5}{2}}}, \\
\hat{\beta} \approx \mathcal{T}_{H} \frac{6.14 \times 10^{12}}{\ln \Lambda_{e i}} \frac{T_{k e V}^{\frac{7}{2}}}{L_{T \mu}^{2} P_{0 G}} \frac{\beta_{\curlywedge}}{\chi_{e}}, \\
\frac{2(g-1)}{5 P_{0}} T \hat{\kappa} \approx \mathcal{T}_{H} \frac{1.84 \times 10^{12}}{\ln \Lambda_{e i}} \frac{T_{k e V}^{\frac{7}{2}}}{L_{T \mu}^{2} P_{0 G}}\left(\kappa_{\perp e}^{c}+\sqrt{\frac{2 m_{e}}{m_{i}}} \kappa_{\perp i}^{c}\right), \\
\hat{\gamma} \approx \mathcal{T}_{H} \frac{4.09 \times 10^{-2}}{\ln \Lambda_{\alpha e}} \mathcal{E}_{\alpha} \frac{T_{k e V}^{\frac{7}{2}}}{L_{T \mu}^{2} P_{0 G}^{2}} \Gamma_{\chi},
\end{gathered}
$$

where $T_{k e V}$ is the fuel temperature in $\mathrm{keV}, P_{0 G}$ is the fuel pressure in Gbar, and $L_{T \mu}$ is the temperature length scale in $\mu \mathrm{m}$.

From these relations, we can see that the Nernst and thermal conductivity terms are very similar in magnitude. Their dependence on $\chi_{e}$ is shown in Fig. 9. This figure illustrates that we expect the largest magnetization growth rates to occur at small values of $\chi_{e}$. It is likely that the cryogenic fuel layers of many MIF schemes will have initially low $\chi_{e}$ values due to the high density, and so, $\chi_{e}$ can increase rapidly in such regions as burn propagates.

Finally, we can take the ratio of the $\alpha$ heating term to the $\alpha-\mathrm{e}$ collisional term to obtain a criterion (which is independent of $\mathcal{E}_{\alpha}$ ) for when the latter term is larger,

$$
T_{k e V}^{6}\left|\Gamma_{\chi}\right|>\frac{\left(L_{T \mu} P_{0 G} \ln \Lambda_{\alpha e}\right)^{2}}{650} .
$$

From this formula, we can, for given values of $\chi_{e}, L_{T \mu}$, and $P_{0 G}$, calculate the temperature above which the $\alpha$-e collisional term is larger. An example of this is shown in Fig. 10.

\section{CONCLUSIONS}

In this work, we have studied the propagation of a subsonic thermonuclear burn wave across a magnetic field in a high beta plasma using an MHD model. We can summarize our findings as follows:

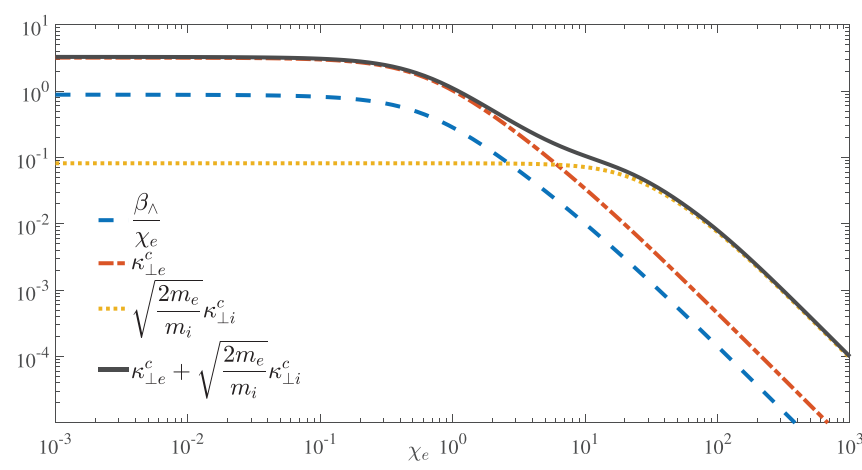

FIG. 9. The variation of the dimensionless thermoelectricity and thermal conductivity coefficients with $\chi_{e}$. For $\chi_{e} \geqslant 6$, ion thermal conductivity is larger than electron thermal conductivity. 


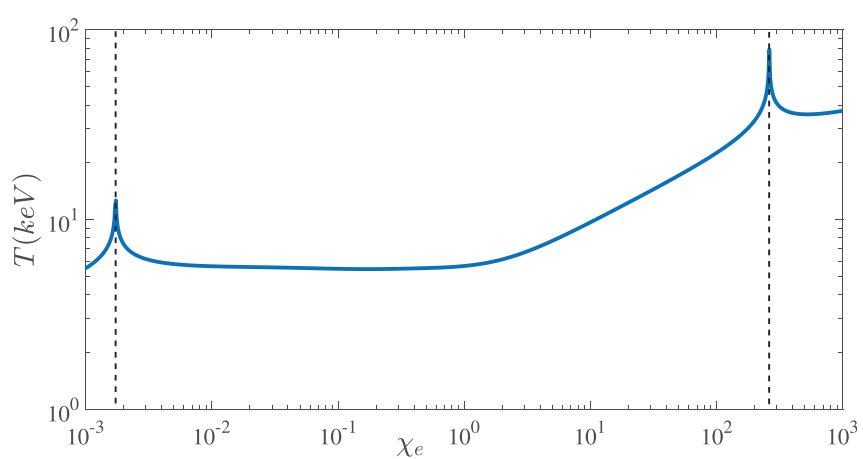

FIG. 10. A plot of the minimum temperature for which the $\alpha-\mathrm{e}$ collisional term dominates the $\alpha$ heating term in the growth rate of $\chi_{e}$ for $L_{T \mu}=10$ and $P_{0 G}=100$. This result may be scaled according to (13) for the other values of $L_{T \mu}$ and $P_{0 G}$. The dashed vertical lines indicate the values of $\chi_{e}$ for which $\Gamma_{\chi}=0$.

i. Ablation of cold fuel due to thermonuclear burn advects the $B$ field from cold to hot fuel at the burn front, while the Nernst and $\alpha-e$ collision terms transport the $B$ field from hot to cold fuel. These combined transport effects confine the $B$ field in the burn front region and cause both compression and rarefaction of $B$

ii. The electron and $\alpha$ Hall parameters, $\chi_{e}$ and $\chi_{\alpha}$, can grow rapidly at the burn front due to $\alpha$ heating, magnetic field transport, and thermal conduction processes. The growth rate at the burn front can be significantly larger than in the hot fuel, causing a self-insulating layer to form at the burn front.

iii. The temperature profile of a magnetized burn front is steeper than the unmagnetized case due to the suppression of thermal conduction. Furthermore, the formation of a self-insulating layer can lead to very steep temperature gradients in localized regions of the burn front.

iv. A magnetized burn front reduces the rate at which cold fuel is burnt due to the self-insulating effect.

These results demonstrate that careful modeling of the $B$ field transport during burn is required to accurately simulate the propagating burn wave and predict the energy gain achieved. However, some of the assumptions in our idealized model prevent us from making quantitative predictions for how $B$ field transport could affect specific MIF schemes.

The isobaric condition restricts us to regimes of subsonic burn (deflagration). Our results prompt the question of whether the formation of a self-insulating layer at the burn front could cause a transition to detonation of the hot fuel. If the burn front is sufficiently subsonic, then the isobaric condition will hold throughout burn, and so, the selfinsulating layer will prevent energy flow from hot to cold fuel until burn is extinguished in the hot fuel. However, if the isobaric condition becomes invalid during heating of the plasma, then the steepening profiles in the self-insulating layer and increasing pressures could lead to the formation of a shock-like burn front corresponding to a detonation regime.

Another aspect of our model requiring further study is geometry. The planar geometry used here is suitable for idealized studies of burn front dynamics since the hot fuel at infinity is unaffected by the cold fuel. It is likely that there will be a more complex interplay between hot fuel and burn front in finite sized targets in spherical or cylindrical geometry. In addition, it is not known how perturbations and multidimensional features at the burn front could affect the formation of a self-insulating layer. Questions such as these will be addressed in future work using integrated simulations.

The results also provide further evidence ${ }^{2,11}$ that an optimum magnetic field strength and profile shape could exist for a given MIF scheme. Such a field would need to provide the correct amount of magnetothermoinsulation during compression and ignition, followed by sufficient confinement of $\alpha$ particles during burn propagation without unnecessary slowing of the burn wave. The strength and shape of this optimum magnetic field and how it can be generated remain open questions.

\section{DATA AVAILABILITY}

The data that support the findings of this study are available from the corresponding author upon reasonable request.

\section{REFERENCES}

${ }^{1}$ J. Lindl, "Development of the indirect-drive approach to inertial confinement fusion and the target physics basis for ignition and gain," Phys. Plasmas 2(11), 3933-4024 (1995).

${ }^{2}$ S. A. Slutz and R. A. Vesey, "High-gain magnetized inertial fusion," Phys. Rev. Lett. 108, 025003 (2012).

${ }^{3}$ J. K. Tong, K. McGlinchey, B. D. Appelbe, C. A. Walsh, A. J. Crilly, and J. P. Chittenden, "Burn regimes in the hydrodynamic scaling of perturbed inertial confinement fusion hotspots," Nucl. Fusion 59(8), 086015 (2019).

${ }^{4}$ G. A. Wurden, S. C. Hsu, T. P. Intrator, T. C. Grabowski, J. H. Degnan, M. Domonkos, P. J. Turchi, E. M. Campbell, D. B. Sinars, M. C. Herrmann, R. Betti, B. S. Bauer, I. R. Lindemuth, R. E. Siemon, R. L. Miller, M. Laberge, and M. Delage, "Magneto-inertial fusion," J. Fusion Energy 35(1), 69-77 (2016).

${ }^{5}$ I. R. Lindemuth and R. C. Kirkpatrick, "Parameter space for magnetized fuel targets in inertial confinement fusion," Nucl. Fusion 23(3), 263-284 (1983).

${ }^{6}$ S. A. Slutz, M. C. Herrmann, R. A. Vesey, A. B. Sefkow, D. B. Sinars, D. C. Rovang, K. J. Peterson, and M. E. Cuneo, "Pulsed-power-driven cylindrical liner implosions of laser preheated fuel magnetized with an axial field," Phys. Plasmas 17(5), 056303 (2010)

${ }^{7}$ M. R. Gomez, S. A. Slutz, C. A. Jennings, D. J. Ampleford, M. R. Weis, C. E. Myers, D. A. Yager-Elorriaga, K. D. Hahn, S. B. Hansen, E. C. Harding, A. J. Harvey-Thompson, D. C. Lamppa, M. Mangan, P. F. Knapp, T. J. Awe, G. A. Chandler, G. W. Cooper, J. R. Fein, M. Geissel, M. E. Glinsky, W. E. Lewis, C. L. Ruiz, D. E. Ruiz, M. E. Savage, P. F. Schmit, I. C. Smith, J. D. Styron, J. L. Porter, B. Jones, T. R. Mattsson, K. J. Peterson, G. A. Rochau, and D. B. Sinars, "Performance scaling in magnetized liner inertial fusion experiments," Phys. Rev. Lett. 125, 155002 (2020).

${ }^{8}$ L. J. Perkins, B. G. Logan, G. B. Zimmerman, and C. J. Werner, "Twodimensional simulations of thermonuclear burn in ignition-scale inertial confinement fusion targets under compressed axial magnetic fields," Phys. Plasmas 20(7), 072708 (2013).

${ }^{9}$ L. J. Perkins, D. D.-M. Ho, B. G. Logan, G. B. Zimmerman, M. A. Rhodes, D. J. Strozzi, D. T. Blackfield, and S. A. Hawkins, "The potential of imposed magnetic fields for enhancing ignition probability and fusion energy yield in indirect-drive inertial confinement fusion," Phys. Plasmas 24(6), 062708 (2017).

${ }^{10} \mathrm{R}$. D. Jones and W. C. Mead, "The physics of burn in magnetized deuteriumtritium plasmas: Spherical geometry,” Nucl. Fusion 26(2), 127-137 (1986).

${ }^{11}$ A. L. Velikovich, J. L. Giuliani, R. W. Clark, and S. T. Zalesak, "Thermonuclear burn wave propagation across an ultrahigh magnetic field," in 2012 Abstracts IEEE International Conference on Plasma Science (2012), p. 4C-5.

${ }^{12}$ L. Biermann, "Uber den Ursprung der Magnetfelder auf Sternen und im interstellaren Raum," Z. Naturforsch, 5, 65 (1950).

${ }^{13}$ C. A. Walsh, J. P. Chittenden, K. McGlinchey, N. P. L. Niasse, and B. D. Appelbe, "Self-generated magnetic fields in the stagnation phase of indirect- 
drive implosions on the National Ignition Facility," Phys. Rev. Lett. 118, 155001 (2017).

${ }^{14}$ A. L. Velikovich, J. L. Giuliani, and S. T. Zalesak, "Magnetic flux and heat losses by diffusive, advective, and Nernst effects in magnetized liner inertial fusionlike plasma," Phys. Plasmas 22(4), 042702 (2015).

${ }^{15}$ A. L. Velikovich, J. L. Giuliani, and S. T. Zalesak, "Nernst thermomagnetic waves in magnetized high energy density plasmas," Phys. Plasmas 26(11), 112702 (2019)

${ }^{16}$ F. García-Rubio, J. Sanz, and R. Betti, "Magnetic flux conservation in an imploding plasma,” Phys. Rev. E 97(1), 011201 (2018).

${ }^{17}$ F. García-Rubio and J. Sanz, "Mass ablation and magnetic flux losses through a magnetized plasma-liner wall interface," Phys. Plasmas 24(7), 072710 (2017).

${ }^{18}$ S. I. Braginskii, "Transport processes in a plasma," Rev. Plasma Phys. 1, 205 (1965).

${ }^{19}$ E. M. Epperlein and M. G. Haines, "Plasma transport coefficients in a magnetic field by direct numerical solution of the Fokker-Planck equation," Phys. Fluids 29(4), 1029-1041 (1986).

${ }^{20}$ W. A. Farmer, C. L. Ellison, and J. H. Hammer, "Linear response of a Hall magnetic drift wave for verification of Hall MHD algorithms," Phys. Plasmas 26(7), 072120 (2019).

${ }^{21}$ N. J. Fisch, "Transport in driven plasmas," Phys. Fluids 29(1), 172-179 (1986).

${ }^{22}$ X.-N. Bai, D. Caprioli, L. Sironi, and A. Spitkovsky, "Magnetohydrodynamic particle-in-cell method for coupling cosmic rays with a thermal plasma: Applications to non-relativistic shocks," Astrophys. J. 809(1), 55 (2015).

${ }^{23} \mathrm{M}$. Sherlock, "Generalized Ohm's law for a background plasma in the presence of relativistic charged particles," Phys. Rev. Lett. 104, 205004 (2010).

${ }^{24}$ B. Appelbe, M. Sherlock, O. El-Amiri, C. Walsh, and J. Chittenden, "Modification of classical electron transport due to collisions between electrons and fast ions," Phys. Plasmas 26(10), 102704 (2019).

${ }^{25}$ M. A. Liberman and A. L. Velikovich, "Distribution function and diffusion of $\alpha$-particles in DT fusion plasma," J. Plasma Phys. 31(3), 369-380 (1984).
${ }^{26}$ H. S. Bosch and G. M. Hale, "Improved formulas for fusion cross-sections and thermal reactivities," Nucl. Fusion 32(4), 611 (1992).

${ }^{27}$ A. S. Richardson, NRL Plasma Formulary (Naval Research Laboratory, 2019).

${ }^{28}$ A. Nishiguchi, T. Yabe, M. G. Haines, M. Psimopoulos, and H. Takewaki, "Convective amplification of magnetic fields in laser-produced plasmas by the Nernst effect," Phys. Rev. Lett. 53, 262-265 (1984).

${ }^{29}$ V. Drean, M. Olazabal-Loumé, J. Sanz, and V. T. Tikhonchuk, "Dynamics and stability of radiation-driven double ablation front structures," Phys. Plasmas 17(12), 122701 (2010).

${ }^{30}$ J. Sanz, R. Betti, V. A. Smalyuk, M. Olazabal-Loume, V. Drean, V. Tikhonchuk, X. Ribeyre, and J. Feugeas, "Radiation hydrodynamic theory of double ablation fronts in direct-drive inertial confinement fusion," Phys. Plasmas 16(8), 082704 (2009).

${ }^{31}$ S. Y. Gus'kov, O. N. Krokhin, and V. B. Rozanov, "Similarity solution of thermonuclear burn wave with electron and $\alpha$-conductivities," Nucl. Fusion 16(6), 957-962 (1976).

${ }^{32}$ O. A. Hurricane, D. A. Callahan, P. T. Springer, M. J. Edwards, P. Patel, K. Baker, D. T. Casey, L. Divol, T. Döppner, D. E. Hinkel, L. F. Berzak Hopkins, A. Kritcher, S. L. Pape, S. Maclaren, L. Masse, A. Pak, L. Pickworth, J. Ralph, C. Thomas, A. Yi, and A. Zylstra, "Beyond alpha-heating: Driving inertially confined fusion implosions toward a burning-plasma state on the National Ignition Facility," Plasma Phys. Controlled Fusion 61(1), 014033 (2019).

${ }^{33}$ A. R. Christopherson, R. Betti, S. Miller, V. Gopalaswamy, O. M. Mannion, and D. Cao, "Theory of ignition and burn propagation in inertial fusion implosions," Phys. Plasmas 27(5), 052708 (2020).

${ }^{34} \mathrm{~V}$. Lobatchev and R. Betti, "Ablative stabilization of the deceleration phase Rayleigh-Taylor instability,” Phys. Rev. Lett. 85, 4522-4525 (2000).

${ }^{35} \mathrm{G}$. E. Vekshtein, "Evolution of magnetic field and anomalous thermal losses in a dense plasma," Sov. Phys. J. Exp. Theor. Phys. 57(2), 317-324 (1983).

${ }^{36} \mathrm{G}$. E. Vekshtein, "Self-similar solutions for the compression of a plasma and a magnetic field by a liner," Sov. Phys. J. Exp. Theor. Phys. 63(3), 528-532 (1986).

${ }^{37}$ A. El-Nadi and M. N. Rosenbluth, "Infinite-limit of the drift instability," Phys. Fluids 16(11), 2036-2037 (1973). 